\title{
Politique
}

\section{Stratégie économique et restructuration de l’État au Canada}

\section{François Houle}

Numéro 3, hiver 1983

La crise des finances publiques au Québec

URI : https://id.erudit.org/iderudit/040418ar

DOI : https://doi.org/10.7202/040418ar

Aller au sommaire du numéro

Éditeur(s)

Société québécoise de science politique

ISSN

0711-608X (imprimé)

1918-6584 (numérique)

Découvrir la revue

Citer cet article

Houle, F. (1983). Stratégie économique et restructuration de l'État au Canada. Politique, (3), 63-87. https://doi.org/10.7202/040418ar d'utilisation que vous pouvez consulter en ligne.

https://apropos.erudit.org/fr/usagers/politique-dutilisation/ 


\title{
Stratégie économique et restructuration de l'État au Canada
}

\author{
François Houle \\ Université d'Ottawa
}

Depuis le gouvernement d'Union et l'abolition des tarifs préférentiels avec la Grande-Bretagne ${ }^{1}$, la bourgeoisie canadienne a toujours été incapable de mettre de l'avant un projet cohérent de développement économique qui aurait permis à la fois l'émergence et le développement d'une bourgeoisie nationale dominante, et l'industrialisation du Canada. Ceci vaut tout autant pour la Politique Nationale de 1879 - qui avait davantage un objectif de finance publique que d'industrialisation - pour la politique de coopération économique sur le plan international de C.D. Howe, pendant et après la Seconde Guerre mondiale, que pour la politique de portes ouvertes des années 1950 et 1960. Toutefois, cette volonté de formuler une stratégie économique pour le Canada a persisté. Les transformations récentes au sein de l'économie et de la bourgeoisie au Canada ont permis, pour ne pas dire exigé, l'élaboration d'une nouvelle stratégie économique.

L'objectif de ce texte est de cerner et d'analyser la stratégie de développement économique que propose le gouverne-

1. À partir de 1846 la Grande-Bretagne abolit progressivement les taux préférentiels avec ses colonies. Rappel des Corn Laws (1846) et du Navigation Act (1849), fin des taux préférentiels sur les importations de bois (1851). 
ment fédéral dans le but d'assurer une relance de l'accumulation et un retour à la prospérité économique. Nous verrons comment cette stratégie s'inscrit dans une tentative de sortie de la crise impliquant la restructuration du rapport salarial dominant et de l'État fédératif au Canada. En dernier lieu, nous discuterons de la crise fiscale afin de démontrer pourquoi celle-ci ne peut-être considérée comme la cause première des restructurations en cours.

\section{Mise en place d'une stratégie industrielle}

Depuis la fin des années 60 et le début des années 70 le gouvernement central a tenté de mettre sur pied, la plupart du temps à la pièce, une nouvelle stratégie de développement industriel propre au Canada. Cependant, l'intégration de l'économie canadienne à l'économie continentale, la structure fédérative de l'État, les règles du marché mondial et les nécessités de la division internationale du travail (D.I.T.) ont grandement limité la marge de manœuvre du gouvernement fédéral. Le Canada se trouvait dans l'impossibilité de planifier son développement économique en raison de la dominante externe et des contradictions politiques internes ${ }^{2}$, les deux se renforçant

2. Au début des années 1970 plusieurs gouvernements provinciaux et différentes fractions de la bourgeoisie refusèrent la remise en question de l'intégration de l'économie canadienne à l'économie continentale. Pour le gouvernement fédéral cette remise en question, par le biais du contrôle des investissements étrangers, apparaissait comme un premier pas vers la canadianisation du secteur manufacturier. Ce qui semblait essentiel pour assurer que le développement économique canadien se fasse dans l'intérêt du Canada. Cette politique fut abandonnée dès 1972, bien qu'un certain contrôle des investissements étrangers fut maintenu (FIRA).

Sur l'effet de la «spécialisation" régionale de l'économie canadienne, voir Garth Stevenson (1979), Unfulfilled Union, Gage publishing, Toronto. G. Bourque, A. Légaré (1979), Le Québec, la question nationale, Petite Collection Maspéro, Paris. A. Dubuc, "Les fondements historiques de la crise des sociétés canadienne et québécoise», in Politiques Aujourd'bui , 7-8, 1978, 29-53. Pour une problématique cherchant à analyser la formation sociale canadienne en insistant sur son unité plutôt que sur les fractions de classes dominantes au niveau des espaces régionaux, voir G. Boismenu (1981), Le duplessisme, Presses de l'Université de Montréal, Montréal. 
mutuellement. Néanmoins, la crise actuelle et la restructuration
mondiale du capitalisme ont permis au gouvernement fédéral de mettre au point une stratégie de développement économique basée sur les méga-projets ${ }^{3}$, essentiellement dans le secteur énergétique. Plus de $85 \%$ des projets dépassant $100 \$$ millions, prévus pour la période allant jusqu'à l'an 2000, sont reliés au secteur de l'énergie 4 .

La présente stratégie industrielle ${ }^{5}$ mise en place à travers le Programme énergétique national $(\mathrm{PEN})^{6}$, les budgets du gouvernement fédéral de 1980 et 1981 , les ententes fédérales-

3. Les premières déclarations sur les méga-projets datent de 1978 alors que A. W. Gillespie était ministre des Mines et Ressources dans le cabinet fédéral.

4. Selon l'inventaire fait par le groupe de travail sur les bénéfices industriels et régionaux des méga-projets pour le compte du ministère de l'Industrie et du Commerce canadien; $86,5 \%$ des méga-projets seront dans le secteur énergétique, 7,7\% dans le secteur des ressources naturelles, 3,1\% dans le secteur manufacturier et 2,6\% dans ceux des transports et de la défense. cf. Major Canadian Projects, Major Canadian Opportunities, Ottawa, juin 1981.

5. On pourrait s'opposer à l'utilisation du terme stratégie industrielle, en ce sens qu'il n'existe aucune politique globale, unifiée et cohérente de développement industriel. S'il est vrai qu'il ne faut pas surévaluer les capacités régulatrices du gouvernement fédéral, il demeure qu'il existe un ensemble de politiques allant dans un même sens. De plus, il est très difficile pour le gouvernement central canadien de mettre officiellement de l'avant une politique industrielle, car par définition cette dernière ne pourrait qu'être coercitive et discriminatoire et elle susciterait de ce fait de multiples oppositions de la part des différentes classes ou fractions et de plusieurs gouvernements provinciaux.

Ian Stewart, sous-ministre au ministère des Finances, reconnait d'ailleurs que le PEN doit être perçu non seulement comme une politique en matière d'énergie, mais aussi comme faisant partie d'une politique économique globale: "The desire of the government to find a solution to the oil problem that was, in its view, equitable was driven, of course, not only by the second world energy shock of 1979-1980 and the progressive insecurity that continuing vulnerability to interruptions in the international sources of supply held for Canada, but also by the government's desire to construct a broad economic policy as well. Having dictated the primary design of the energy solution, it then became imperative to find a mechanism for putting that energy solution into might be called wise economic policy for the country... " lan Stewart, "Ottawa and the Post-Keynesian World", in D. Crane (ed.) 1981. Beyond the Monetarists, Canadian Institute for Economic Policy, 92.

6. Ministère de l'Énergie, des Mines et des Ressources, Le Programme énergétique national, Ottawa, 1980. 
provinciales sur le pétrole et la gaz (automne 1981) et les huit projets de loi sur l'énergie votés par le Parlement durant la dernière session (première session, trente-deuxième législature), vise à centrer le développement économique canadien sur le secteur énergétique. L'avenir du secteur industriel canadien dépend presqu'exclusivement du développement des moyens énergétiques.

L'échec récent de plusieurs méga-projets qui ont été abandonnés ou remis à plus $\operatorname{tard}^{7}$ démontre la fragilité de la stratégie fédérale dans la présente conjoncture de crise. Toutefois, ce qui nous intéresse ici ce ne sont pas les causes économique et politique de ces échecs mais bien l'analyse à moyen et à long terme de la stratégie économique canadienne. On pourrait essayer de voir dans quelle mesure le programme énergétique national ${ }^{8}$, les politiques monétaires et fiscales, le nationalisme canadien et la conjoncture économique mondiale sont responsables de ces abandons, mais il nous apparaît plus important de tenter de saisir les objectifs globaux du gouvernement fédéral définissant ce que devrait être l'économie canadienne dans le nouvel ordre économique mondial qui possiblement émergera de la crise.

La stratégie industrielle fédérale indique que l'industrialisation canadienne se fera principalement en amont du produit générateur. Par conséquent, les moyens énergétiques, d'intrants

7. Les projets Cold Lake, Alsands et le gazoduc de l'Alaska ont été arrêtés et remis à plus tard, c'est-à-dire lorsque les possibilités de profit apparaîtront satisfaisantes à l'entreprise privée, que le gouvernement fédéral aura modifié sa politique fiscale et qu'il aura atténué ses velléités de canadianisation du secteur de l'énergie.

8. Pour une excellente analyse du PEN en rapport avec la bourgeoisie canadienne et à la crise fiscale du gouvernement fédéral, voir L. Pratt, «Energy: The Roots of National Policy ", Studies in Political Economy, no. 7, Winter 1982, 27-59.

Pour J. Laxer le PEN marque un point tournant, à la fois dans la politique économique canadienne et dans l'érosion des pouvoirs du gouvernement central. Ce qui serait, selon l'auteur, très positif pour l'avenir du Canada. J. Laxer (1981), Canada's Economic Strategy, McClelland and Stewart, Toronto. 
qu'ils étaient pour les sections des moyens de production et des moyens de consommation, se situeront dorénavant en aval d'une partie de la section des moyens de production tout en demeurant, théoriquement, en amont des deux sections. Il semble s'agir en fait d'une restructuration visant à concentrer le développement du secteur de la fabrication au niveau des moyens de production et partant de recentrer le régime d'accumulation autour de la section I et des moyens énergétiques ${ }^{9}$.

Cette stratégie industrielle qui vise à définir les nouveaux lieux d'accumulation pose plusieurs problèmes. Premièrement, le gouvernement fédéral en mettant l'accent sur les moyens énergétiques suppose que ces derniers vont entrer de plain-pied dans le nouveau régime d'accumulation ${ }^{10}$. Si cette hypothèse est réaliste pour les formations sociales avancées, elle demeure pour le moins problématique en ce qui a trait aux pays de la périphérie. Pour en arriver à une telle transformetion dans les pays périphériques et semi-périphériques producteurs de

9. Cette volonté de recentrer le secteur de la fabrication en amont du secteur des ressources et autour des industries de haute-technologie est en accord avec le PEN. A.J. Olson, alors qu'il était ministre d'État au Développement économique, affirmait: «... The opportunities in resources provide the promise fot growth and a renewal in Canadian manufacturing... As the number of sellers in the world market involved in making certain kinds of labour intensive goods increases, we have the opportunity to concentrate on and specialize in those things we can produce best...» p. 3

Les entreprises liées au secteur des ressources et à la haute-technologie, selon le ministre Olson, bénéficient d'un avantage pour tenter une percée au niveau du marché mondial. Source: "Priorities for Federal Government Economic Development and Expenditures to the Mid-1980's ".

10. Il est impossible de définir quelles seront les bases du nouveau régime d'accumulation. Pour J.H. Lorenzi. O. Pastre et J. Toledano (1980), La crise au XXème siècle, Economica, la sortie de crise semble résider dans l'approfondissement de l'accumulation intensive, relance basée sur un redémarrage des gains de productivité, la transformation des formes de consommation et d'une partie du travail improductif en travail productif. Par contre pour D. Dufour la relance de l'accumulation se fera à partir de l'émergence du régime d'accumulation progressive, lequel consisterait en une juxtaposition de l'accumulation extensive et intensive et s'appuierait sur un accroissement de l'intervention de l'État dans l'économie et la mise en place d'une N.D.I.T. D. Dufour (1980), "Transformations de l'économie mondiale et crise de la régulation étatique», in État et Régulations, Presses Universitaires de Lyon, Lyon. 
moyens énergétiques, le procès de travail dans ce secteur devra être modifié, ce qui nécessitera non seulement des transformations sociales importantes (desquelles émergeront de nouvelles luttes) mais aussi: soit des modifications de leur structure industrielle, soit une plus grande intégration de ces pays aux pays industrialisés. Dans ce dernier cas le résultat ne ferait qu'aggraver leur développement dépendant et leur intégration au marché mondial en accélérant la relocalisation de branches industrielles. En outre cette stratégie fédérale implique que le régime s'accumulation intensive autocentrée va sortir de sa crise sur des bases permettant l'intégration de cet intrant que sont les ressources énergétiques traditionnelles.

Enfin, cette politique présuppose que la D.I.T. des années 60 entre le tiers monde exportateur de matières premières et de produits énergétiques et les pays capitalistes avancés ne renaîtra pas sous les pressions industrielles et financières causées par l'extension d'un rapport salarial dans certains pays périphériques ou semi-périphériques exportateurs de ressources énergétiques. La difficulté pour les pays de l'OPEP de diminuer la production de pétrole afin de soutenir les prix résulte, dans une large mesure, de l'impératif pour certains pays producteurs d'obtenir des sommes d'argent suffisantes pour financer leur tentative d'industrialisation et pour faire face à leur endettement sur les marchés internationaux de capitaux. L'exemple du Nigéria est très éloquent sur ce point.

Un second problème lié à la stratégie économique fédérale réside dans sa tentative de concentrer l'industrialisation en amont des moyens énergétiques; ce qui aura pour résultat de centrer le secteur industriel sur la section des moyens de production tout en la sur-spécialisant. Un tel développement soulève dans toute son ampleur la question de l'intégration du secteur de la fabrication canadienne à l'économie continentale. La faiblesse de l'innovation technologique et de la recherche au 
Canada par rapport à la technologie étatsunienne (et de certains autres pays industrialisés), de même que la forte présence de filiales d'entreprises multinationales ne garantissent aucunement que le développement d'industries et de services en amont des produits énergétiques se fera au profit de la bourgeoisie nationale. Pour obtenir un tel résultat l'État canadien devra envisager toute une série de mesures coercitives précises pour non seulement limiter le propriété étrangère dans le secteur des ressources mais également pour s'assurer que le développement industriel en amont profitera davantage aux entreprises canadiennes. Une telle politique est essentielle pour le succès de la stratégie fédérale. Des règles trop permissives risquent de produire les mêmes résultats que ceux engendrés par la Politique Nationale (1879); c'est-à-dire l'implantation de filiales desservant uniquement le marché local. Une telle orientation industrielle remettrait en cause un autre objectif du gouvernement fédéral, soit le développement d'industries et de services «sous-traitants» du secteur énergétique, qui viseraient à s'approprier une partie du marché mondial.

Malgré l'ampleur des sommes investies, un marché aussi spécialisé que la fabrication des moyens de production pour le secteur énergétique, demeure limité au niveau national et le seul moyen d'assurer la viabilité de ces entreprises est d'en diminuer les coûts par des économies d'échelle. Les luttes interimpérialistes pour la conquête du marché mondial seront cependant très vives. L'exemple de l'industrie nucléaire canadienne en dit long sur la nécessité et les difficultés de conquérir une part du marché mondial. Même l'aide soutenue de l'État n'a pas permis de surmonter ce problème vital.

Cette vision qu'a le gouvernement fédéral de l'avenir du secteur de la fabrication démontre l'acceptation de certaines transformations au sein de l'économie mondiale. Aucun effort véritable n'est axé sur le maintien et le développement du sec- 
teur de la production des marchandises banalisées de consommation de masse. De plus, la section des moyens de production est perçue comme participant d'emblée à une économie capitaliste mondiale émergente. En fait, le Canada veut jouer la carte de la spécialisation économique sans réaliser jusqu’à quel point une telle stratégie placera l'économie canadienne dans une situation de dépendance face au marché mondial, tout en la soumettant davantage aux aléas de la conjoncture économique internationale et à la concurrence des formations sociales hégémoniques.

Cette stratégie économique en plus d'avoir à surmonter les problèmes ci-haut mentionnés, nécessite une restructuration à la fois de l'État canadien et du rapport salarial dominant. Seul un certain nombre d'auteurs, que l'on pourrait qualifier de keynésiens de gauche, croient en la possibilité d'une relance à partir des mêmes bases d'accumulation et du maintien du rapport salarial fordiste. Selon eux le problème des économies occidentales repose sur une trop faible productivité. Une révolution technologique pourrait par conséquent permettre de dégager une super-productivité entraînant, d'une part, une diminution de la force de travail productive et, d'autre part, l'augmentation du taux d'exploitation, ce qui rendrait possible le maintien du rapport salarial fordiste en accentuant le développement du secteur tertiaire et de l'État-providence. Cette analyse dénote non seulement une vision étriquée de la crise mais elle présuppose que cette dernière n'en est pas une puisqu'il ne s'agit pas d'une crise structurelle mais d'un simple problème de dysfonctionnement. Cette thèse s'apparente à celle de $\mathbf{M}$. Aglietta [1976, Régulations et crises du capitalisme, CalmannLevy, Paris] pour qui la crise de régulation est en partie dûe à un blocage de la section I, laquelle n'a pas réussi à accroître de façon durable la productivité de la section des moyens de communication. 
Une brève analyse de la crise et du rapport salarial fordiste montrera en quoi la stratégie industrielle fédérale n'est qu'un des éléments actuellement mis en place pour rendre possible une relance de l'accumulation capitaliste au Canada en l'intégrant davantage au marché mondial.

\section{Restructuration du rapport salarial fordiste}

La présente crise que traversent les formations sociales capitalistes avancées peut-être qualifiée de crise de l'accumulation intensive autocentrée. Elle origine de deux types de contradictions inhérentes au stade actuel d'accumulation, le premier, interne au procès de production immédiat est lié à la hausse de la composition organique du capital et à l'épanouissement des possibilités de «révolutionarisation» au niveau du procès de travail. Quant au second, il se situe à l'intérieur même de la dynamique du rapport salarial fordiste instauré au Canada surtout à partir de 1935. C'est-à-dire essentiellement l'application par le niveau fédéral de l'État canadien des théories keynésiennes et des conclusions du rapport Beveridge ${ }^{11}$ quant aux rôles de l'État comme régulateur de l'économie de marché et soutien des modes de consommation.

Le rapport salarial fordiste peut-être considéré comme la matérialisation du régime d'accumulation intensive et d'un rapport de forces, en ce sens qu'il caractérise le primat de la

11. En Grande-Bretagne, Sir William Beveridge publia un rapport en novembre 1942 identifiant les problèmes qui devaient être solutionnés pour assurer la reconstruction après la seconde guerre mondiale. Ce rapport inspira plusieurs études au Canada dont les principales sont:

L. C. Marsh, Report on Social Security for Canada, 1943, Reprint by University of Toronto Press, Toronto, 1975.

Department of Reconstruction, Employment and Income with Special Reference to the Initial Period of Reconstruction, Ottawa, 1945.

Dominion-Provincial Conference on Reconstruction, Proposals of the Government of Canada, Ottawa, 1945. 
section des biens de consommation sur la section des biens de production, l'approfondissement du procès de travail tayloriste, l'émergence d'un double mode de consommation et enfin les transformations des fonctions historiques de l'État tant au niveau de la gestion que de l'entretien/reproduction de la force de travail, que cette dernière soit salariable ou non ${ }^{12}$.

La crise actuelle est donc une crise organique où sont remis en question non seulement les rapports capital/travail dans une tentative d'accentuation de la soumission réelle et formelle des travailleurs/travailleuses, mais aussi l'ensemble de la dynamique d'accumulation liée au procès de valorisation ${ }^{13}$ et à la résolution temporaire des contradictions entre les rapports de production et d'échange.

La crise ne découle pas des hausses de salaire des travailleurs ${ }^{14}$ mais $1^{\circ}$ de l'épuisement du modèle de croissance et $2^{\circ}$

12. Pour une discussion en profondeur de l'État et du rapport salarial fordiste, cf. François Houle, Rapport salarial, Crise et État; essai de théorisation sur la crise des pays du centre, miméo, département de science politique, Université d'Ottawa, Mars 1982, 60 pages.

En bref, nous définissons le rapport salarial comme un rapport de soumission/adaptation/intégration. Par conséquent, le rapport salarial fordiste est la caractérisation particulière du rapport salarial au stade de l'accumulation intensive. Cette définition, tout en s'inspirant des théories de la régulation, s'en démarque sur un point précis, à savoir qu'il s'agit d'un rapport essentiellement social sans aucune référence directe à son aspect monétaire.

Pour une excellente présentation du régime d'accumulation intensive, voir A. Lipietz (1979), Crise et inflation, pourquoi?, Maspéro, Paris.

13. Marx, Karl (1971), Un chapitre inédit du capital, coll. 10/18, Paris, Pages 78, 122, 145.

14. Thèse soutenue par les néo-ricardiens tels: Glyn, A., et Sutcliffe, R. (1972), British Capitalism, Workers and the Profit Squeeze, Penguin Brooks, London. De toute façon, il est impossible de mesurer la rentabilité sociale du capital à partir du rapport entre la croissance du salaire direct et l'accroissement de la productivité. Il n'y a pas de passage simple, même dans l'analyse concrète, entre la loi de la valeur et une théorie des prix.

Plusieurs auteurs, dont J.-G. Lacroix, dans "Quel socialisme opposer à l'attaque du Capital ", in Les cabiers du socialisme, no. 9, hiver 1982, 155-187, voient les luttes menées par les travailleurs contre la perte de leur pouvoir d'achat comme «le point d'inflexion dans la régulation sociale de l'État providence», p. 155. S'il est certain 
des luttes du travailleur collectif pour soi ${ }^{15}$ aux niveaux économique (d'abord et avant tout au niveau du procès de travail) et politique (principalement lutte des mouvements ouvrier et populaire pour le maintien d'acquis au niveau du salaire indirect). Les hausses importantes de salaire ne furent gagnées par les travailleurs canadiens qu'après le début de la crise (période 1974-1977). Elles ne constituent qu'une tentative de leur part pour se prémunir contre l'inflation qui fut un des principaux moyens du capital pour lutter contre la crise en en faisant porter le poids aux travailleurs ${ }^{16}$.

S'il est certain qu'une sortie de crise valable pour le capital exige une défaite majeure de la classe des salariés en impulsant une hausse importante et durable du taux d'exploitation, ceci n'est nullement suffisant. Une sortie de crise commande aussi la restructuration du régime d'accumulation donc du rapport salarial dominant et de l'État.

Le capital pour assurer sa reproduction élargie s'appuie non seulement sur le procès de production de plus-value (à

que ces luttes ont accéléré l'approfondissement de la crise de l'accumulation intensive, la crise était déjà présente dans l'inflation.

Il ne faudrait pas non plus sous-estimer ces luttes en les assimilant à un simple impact conjoncturel; on oublierait alors que l'indexation des salaires fut une défaite pour le capital dans sa tentative de redistribution de la valeur ajoutée.

15. Pour une analyse du processus de décomposition/recomposition du travailleur collectif, cf. C. Palloix (1981), De la socialisation, coll. Interventions en économie politique, P.U.G. - Maspéro, Paris.

16. D'ailleurs, même selon les statistiques gouvernementales, le revenu personnel réel des travailleurs diminue depuis 1978 et pourtant la crise continue de s'approfondir. La situation est encore plus pénible pour la force de travail non salariée et/ou non salariable qui ne compte que sur les paiements de transfert gouvernementaux. Ministère des Finances (1981), Revue économique, tableau II, Ottawa.

$\grave{A}$ cette tentative de faire porter la crise sur le salaire direct des travailleurs/travailleuses au moyen de l'inflation, s'ajoute la fiscalité. Ainsi au Québec, alors que le taux effectif d'imposition était en 1972 de 7,7\% pour la moyenne des revenus (impôt payé de $\$ 483$ pour la moyenne des revenus de $\$ 6,031$ ), le taux d'imposition était de $10,5 \%$ en 1979 (impôt payé $\$ 724$ pour la moyenne des revenus de $\$ 6,920$ - ces derniers chiffres sont en dollars de 1972). Source: Ministère du Revenu du Québec, Statistiques fiscales des particuliers du Québec, pour l'année. 
l'intérieur du procès de valorisation) mais aussi sur l'établissement des conditions nécessaires de sa réalisation. Cette seconde exigence ne peut aller à l'heure actuelle, dans la plupart des formations sociales avancées, sans une restructuration du rapport salarial fordiste, elle se double pour le Canada d'une restructuration de l'État allant dans le sens d'une forte centralisation.

La logique du rapport salarial fordiste a impliqué dans une large mesure la croissance de l'État, c'est-à-dire une transformation de ses fonctions historiques ${ }^{17}$. Ainsi, l'émergence et le développement de l'État-providence ne peut être compris simplement ni comme conséquence de la phase expansionniste ni comme résultat de la lutte de classe au niveau politique. Cette forme étatique est à la fois cause et conséquence de l'accumulation intensive. Donc, la remise en question de l'État providence fait partie de la restructuration du rapport salarial.

Le gouvernement fédéral tente depuis 1975 une remise en question systématique de certaines de ses interventions aux niveaux des «services» et des paiements de transfert. La première étape fut celle des modifications législatives au niveau des paiements de transfert aux particuliers visant à en modifier les conditions d'admission (assurance-chômage) et à en restreindre l'universalité par le biais de la fiscalité. Quant aux politiques en matière de santé, d'éducation post-secondaire et d'hospitalisation, leur remise en question fut relativement lente et ce n'est que depuis quelques années qu'on en sent les effets. Ce retard provient de la nature de l'État fédératif çanadien et de la rigidité des accords fiscaux. Ainsi fallut-il attendre

17. Pour la distinction entre forme minimale et forme historique de l'État capitaliste, cf. François Houle, op. cit. Cette distinction s'inspire en partie de celle établie par J. Hirsch entre dérivation logique et dérivation de fonction (1975), "Éléments pour une théorie matérialiste de l'État", in L'État contemporain et la marxisme, Critiques de l'économie politique, Maspéro, Paris. 
jusqu'en 1977 pour que le gouvernement fédéral mette en branle un premier changement important. Ce n'est qu'avec la renégociation des accords fiscaux en 1981-1982 que se révèle la stratégie centralisatrice fédérale. Après avoir lié la croissance du financement de ces "services" à celle du produit national brut (PNB) et avoir laissé les provinces diminuer leur part de financement $^{18}$, le gouvernement fédéral les accuse maintenant d'avoir sabré dans ces services. Il en profite pour justifier son intrusion dans des sphères de juridiction provinciale afin d'établir des normes nationales dans le domaine de la santé et forcer les provinces à restructurer l'enseignement post-secondaire en fonction des besoins du marché du travail ${ }^{19}$.

Ce recul quant aux politiques sociales s'accompagne d'une volonté de l'État de transformer le procès de travail dans les secteurs public et para-public en introduisant quelques éléments du taylorisme, particulièrement la déqualification et la suppression des temps morts, et par l'introduction rapide de nouvelles technologies: micro-informatique, bureaucratique et robotique $^{20}$.

18. Avant les accords de 1977 , le financement de ces programmes à frais partagés se faisait 50/50. En 1977, le fédéral fit accepter comme règle de financement que sa contribution serait dorénavant augmentée proportionnellement au PNB, permettant ainsi aux provinces de diminuer leur part de financement sans craindre de voir la part fédérale diminuer d'autant. Il y avait quatre éléments dans la contribution fédérale de FPE: des paiements de base en espèces, des transferts d'impôts, de la péréquation pour les provinces dont les rendements fiscaux sont inférieurs à la moyenne nationale et des réajustements provisoires. Les contributions se chiffraient à $\$ 443$ dollars par habitant en 1981-1982. Les arrangements fiscaux dans les années quatre-vingt, Ministère des Finances, Ottawa, novembre 1981.

19. La proposition fédérale pour le F.P.E. (1982-1987) vise, tout en maintenant leur croissance au PNB, à diminuer directement les paiements en espèces aux provinces riches et à abroger la garantie de recette. Cette dernière coupure, même si elle ne diminue pas le FPE, diminuera les revenus de l'ensemble des provinces. Cf. idem, note 18 .

20. Pour une discussion de l'innovation technologique en rapport avec l'organisation du travail et au marché de l'emploi, voir Céline St-Pierre, «La crise, le travail, et le travail en crise», in Le Devoir, jeudi 4 février 1982, p. 18. 
Ces attaques contre les conditions de travail et de vie des travailleurs/travailleuses touchent non seulement les multiples groupes minoritaires, comme les femmes, les jeunes, les chômeurs, les assistés sociaux, mais aussi les travailleurs à pleintemps ayant bénéficié historiquement d'une certaine sécurité d'emploi. À cette décroissance de l'État-providence, et non de l'État comme tel, s'ajoute une détérioration des possibilités revendicatrices du mouvement ouvrier. Qu'il s'agisse des multiples tentatives plus ou moins réussies de tripartisme et de concertation ou des législations obligeant indirectement les syndicats ouvriers à participer à la gestion de la classe ouvrière sur une base tripartite, l'ensemble de ces mesures ont pour but d'accentuer l'intégration du mouvement ouvrier à l'idéologie libérale et à limiter de la sorte son rôle de lieu et d'instrument spécifique de lutte, d'opposition et de contestation des rapports socio-économiques capitalistes. Lorsque ces mesures ne suffisent pas pour faire accepter aux salariés une restructuration du rapport salarial, l'État a recours à l'appareil répressif (le judiciaire et la police) pour infliger des défaites aux travailleurs/travailleuses ou invalider quelques-uns de leurs gains ${ }^{21}$.

Toutes ces interventions des gouvernements fédéral et provinciaux ont pour objectif la restructuration du rapport salarial fordiste en limitant une certaine croissance de l'État, en remettant en cause les modes de consommation et finalement en transformant la gestion/reproduction de la force de travail salariée. Cette restructuration a des effets sur les salariés et bien entendu sur la population non-salariée et/ou nonsalariable. Ce rétrécissement du rapport salarial approfondit la crise et risque de déboucher non pas sur une restructuration du

21. Il peut s'agir de lois spéciales de retour au travail, d'injonctions, de lois enlevant ou limitant strictement le droit de grève, de contrôle ou de gel des salaires, de menaces de réouverture des conventions collectives afin de diminuer ou d'éliminer certains bénéfices marginaux. 
régime d'accumulation intensive mais sur l'effondrement de ce dernier. Les raisons motivant l'élimination du rapport salarial fordiste ne doivent pas être saisies uniquement à partir de la crise mais en tenant compte également de la politique industrielle, de la relocalisation et du recentrage des lieux d'accumulation su Canada.

En basant l'avenir de l'accumulation sur les moyens énergétiques et le secteur de la fabrication en amont de ceux-ci, on revient à une situation similaire à celle du régime d'accumulation extensive où le moteur de l'accumulation était en premier lieu la section des moyens de production. La forme dominante de production de plus-value demeurera cependant la plus-value relative. L'édification d'un tel type d'accumulation au Canada facilitant l'intégration de l'économie canadienne à une économie capitaliste mondiale restructurée, respecterait ce qui semble être les tendances de la nouvelle division internationale du travail et ne requérerait aucunement le maintien du modèle de croissance actuel, donc le maintien du rapport salarial fordiste. En fait, son élimination, ou tout au moins son rétrécissement, est un pré-requis pour que l'économie canadienne se soumette aux exigences du marché mondial. Pour maintenir le rapport salarial fordiste l'État devrait accaparer une part importante de la plus-value, ponction qui aurait pour résultat de retarder la restructuration industrielle et par la suite de mettre en péril la place sur le marché mondial des moyens énergétiques canadiens et de quelques branches de la section des moyens de production en amont de ceux-ci. La question du non-maintien du rapport salarial fordiste apparaît donc inséparable de la crise et de la restructuration du régime d'accumulation en rapport avec la division internationale du travail ${ }^{22}$.

22. Pour maintenir le rapport salarial fordiste au Canada il faudrait que le gouvernement fédéral cherche à préserver la dynamique relationnelle entre l'extraction de la plus-value relative, en insistant sur la productivité, le renouvellement des modes de 


\section{Centralisation de l'État et concentration des pouvoirs}

Il est probable que l'ensemble de ces transformations radicales nécessite une centralisation de l'État canadien et une concentration des pouvoirs au niveau de l'appareil fédéral. Selon le gouvernement fédéral, la mise en application de sa politique industrielle et du néo-interventionnisme ${ }^{23}$ ne pourra réussir sans un retour au fédéralisme centralisé de l'aprèsguerre. Pour mettre l'accent sur la mission économique au détriment de la mission sociale, pour contrôler les moyens énergétiques et en planifier les retombées industrielles, pour faciliter la concentration-centralisation du capital canadien et pour soutenir l'internationalisation du capital monopoliste canadien, et d'une partie du capital canadien non monopoliste ${ }^{24}$, le niveau fédéral de l'État canadien doit d'abord en arriver à diminuer les pouvoirs des provinces et de certaines fractions de la bourgeoisie, et à vaincre les résistances et les oppositions des régionalismes économico-politiques.

Cette volonté de centralisation du fédéralisme canadien se perçoit dans le rapatriement de la constitution et la réforme

consommation de masse et collective, et la structuration de l'État. Ceci ne semble aucunement être les objectifs du gouvernement central, et, de plus, une telle politique comme moyen de relancer l'accumulation serait en contradiction avec la mondialisation de la production et de l'échange. Seules les formations sociales hégémoniques peuvent espérer éviter les obligations de spécialisation et «négocier» les conditions de leur participation à l'internationalisation du capital.

23. Le néo-interventionnisme du gouvernement canadien s'appuie sur les théories du «supply-side» mais sans retrait ou décroissance de l'État. En effet, à partir de l'idéologie du nationalisme économique le gouvernement fédéral désire s'impliquer davantage dans l'accumulation et la planification économique en coupant dans l'enveloppe budgétaire des affaires sociales au profit de sa mission économique. Cf. Cy Gonick, «The New Supply Side Economics», in Canadian Dimension, vol. 15, no 4, février 1981 .

24. L'internationalisation du capital productif a longtemps été considérée comme allant de pair avec le développement des monopoles. Certaines recherches récentes démontrent que ce type d'internationalisation est aussi le fait de capitaux non monopolistes. Voir Olle, W. Schoeller, W., «Direct Investment and Monopoly Theories of Imperialism », Capital and Class, 16, Spring 1982, 41-60. 
constitutionnelle ${ }^{25}$, et de surcroît dans la création de PétroCanada, dans le refus de céder les droits miniers sous-marins aux provinces, dans la politique de canadianisation, dans les accords fiscaux, dans la centralisation des interventions du fédéral dans les régions et dans la création de la Commission d'enquête sur l'union économique.

En outre, on constate depuis le milieu des années 1970, chez le gouvernement fédéral, une volonté de concentrer les pouvoirs aux dépens du Parlement, des ministères et d'une partie du cabinet ${ }^{26}$. Cette volonté s'est concrétisée dans la réorganisation du bureau du Premier Ministre (BPM), du bureau du Conseil privé $(\mathrm{BCP})$ et des comités du cabinet (principalement l'importance prise par le comité des Priorités et de la Planification) ${ }^{27}$. Cette concentration du processus de prise de décision politique, administrative et financière a eu récemment comme corollaire une restructuration des ministères à vocation économique et du ministère des Affaires extérieures ${ }^{28}$.

25. Sur le rapatriement, voir D. Brunelle, «Économie politique et rapatriement ", in Les cabiers du socialisme, no 8, automne 1981, 54-80.

26. Dans un discours en 1975, M.P. Pitfield affirme: «Ces dernières années, la double recherche, au sein du gouvernement fédéral, d'une efficacité et d'un rendement accrus et d'une plus grande influence du politique a progressé au rythme des efforts déployés pour mieux définir les objectifs et pour faire en sorte que les programmes du gouvernement soient davantage conformes aux objectifs fixés". M.P. Pitfield, "Le gouvernement des années 80 , techniques et instruments d'élaboration de la politique fédérale», discours à la Conférence Annuelle de l'Institut d'Administration Publique du Canada, miméo., Bureau du Conseil privé, Ottawa, 3 septembre 1975 , p. 6.

27. Pour une présentation des comités du cabinet et des enveloppes budgétaires, voir Bureau du Conseil privé, "Le système de gestion des politiques et des dépenses», Ottawa, mars 1981. Pour la réorganisation du BPM et du BCP, voir Richard J. Van Loon, «Kaleidoscope in Grey: The Policy Process in Canada», in M. S. Whittington and G. Williams eds., (1981), Canadian Politics in the 1980's, Methuen Publications.

28. La restructuration des ministères à vocation économique s'est faite par étape: en 1978 Robert Andras fut nommé président d'un comité pour le développement économique, mais sans une centralisation des budgets et la création d'un ministère avec le personnel nécessaire, il s'avéra difficile de planifier le développement économique. En mai 1979, Joseph Clark mit en application le système d'enveloppes 
Ces processus de centralisation de l'État et de concentration des pouvoirs ont pour finalité de donner au gouvernement fédéral les moyens d'appliquer son néo-interventionnisme et sa stratégie de développement économique. Ces tentatives reçoivent l'appui du capital monopoliste canadien et celui désireux de s'internationaliser. Par contre, les fractions du capital qu'une telle «sortie de crise» menace de marginaliser ou même d'éliminer, se dressent contre l'ensemble de la politique fédérale. Cette opposition se retrouve aussi chez certains gouvernements provinciaux et dans une partie du mouvement ouvrier canadien.

Il est impossible d'évaluer les chances de succès de l'ensemble de ces tentatives de restructuration car elles déclenchent des luttes de résistance importantes et il serait aléatoire de préjuger des résultats des oppositions entre les classes et fractions de classe. En fait, tout dépend du rapport de forces qui s'établira entre les différentes fractions de la bourgeoisie et de l'action/non-action du mouvement ouvrier canadien.

Or le gouvernement fédéral semble connaître une certaine réussite dans la restructuration de son appareil politique et de la fédération canadienne ${ }^{29}$, mais il en va tout autrement de sa

budgétaires et créa le Ministère d'État pour le Développement économique. En 1980, P.E. Trudeau conserva le même système avec un ministre d'État au Développement économique, un comité du cabinet chargé du développement économique et de contrôler l'enveloppe budgétaire pour l'ensemble de la mission économique.

Une autre étape importante fut l'abolition du ministère de l'Expansion économique régionale et la fusion de certaines de ses directions avec le ministère de l'Industrie et du Commerce (MIC). De plus la division du commerce extérieur (de l'ancien MIC) fut transférée au ministère des Affaires extérieures auquel on confia le rôle de mettre l'accent sur la promotion des produits canadiens sur le marché mondial.

29. D. Brunelle, op. cit., note 25 , a raison de voir dans la Charte canadienne des droits et libertés des visées centralisatrices remettant en question le compromis et l'équilibre précaire entre les différentes fractions de la bourgeoisie. Toutefois, cette Charte vise tous les régionalismes (et non seulement la nation québécoise) et les rapports de classe à tous les niveaux de l'État canadien. Enfin rappelons que la réforme constitutionnelle n'est qu'un des éléments visant à mettre fin à "l'érosion" des pouvoirs du gouvernement central. 
stratégie économique. Indéniablement une stratégie basée sur les moyens énergétiques n'a pas de chance réelle de succès dans la présente conjoncture de crise. Toutefois, devant la possibilité de l'émergence d'une nouvelle économie capitaliste mondiale et d'une relance de l'accumulation capitaliste, une spécialisation de l'économie canadienne est pour la fraction hégémonique du capital l'unique choix valable. Car seule une telle stratégie peut rendre possible et soutenir l'internationalisation du capital canadien tout en respectant les exigences de la division internationale du travail et des transformations du marché mondial (accentuation de la libéralisation des échanges accompagnant une plus grande délocalisation de la production) ${ }^{30}$.

\section{Le problème de la crise fiscale}

Avant de terminer nous voudrions aborder rapidement la question de la crise fiscale. Nous avons tenté dans ce texte d'esquisser la logique qui unit la stratégie économique du gouvernement central et les tentatives de restructuration du rapport salarial et de l'État. Cependant, pour plusieurs, les transformations au sein du rapport salarial et de l'État fédératif canadien sont essentiellement le résultat de la crise fiscale. L'hypothèse est que le gouvernement fédéral et plusieurs gouver-

30. La profondeur de la présente crise, les revendications du mouvement ouvrier et de certaines fractions de la bourgeoisie obligèrent le gouvernement central à un retour à un certain protectionisme - négociation d'un quota sur les importations de voitures japonaises et réimposition d'un quota sur les importations de chaussures fabriquées à l'étranger - Le gouvernement central demeure toutefois partisan de la libéralisation des échanges et des derniers accords du GATT de 1979. Mark MacGuigan affirme par exemple: "It is our stake in an open and stable international economic system that has driven home to us the importance of encouraging fuller participation of developing countries in that system... The mutual gains that trade can have to developed and developing countries are not always evident to the unemployed automobile or textile worker, ... But in these days of severe global economic difficulties, a return to the policy of half a century ago would serve none of us. " The Citizen, August 11, p. 9. 
nements provinciaux sont tellement obnubilés par l'ampleur des déficits, qu'ils ne font que chercher, par tous les moyens, à diminuer leurs dépenses et à augmenter leurs revenus ${ }^{31}$.

À croire le discours des hommes politiques et des ténors de la bourgeoisie, le développement de l'État-providence a engendré la crise fiscale. Ce qui justifie les coupures draconiennes dans les programmes sociaux et les paiements de transfert. Quelques-uns vont même jusqu'à affirmer que la crise fiscale est un des éléments qui a provoqué la crise des économies capitalistes ${ }^{32}$.

Quant à nous, l'hypothèse que nous comptons présenter ici est que la crise fiscale, quoique renforçant et mettant à l'avant-plan la nécessaire restructuration du rapport salarial fordiste, doit être analysée comme faisant partie de la crise de l'accumulation intensive. Dès lors, si la crise fiscale n'est pas extérieure à la présente crise, sa résolution complète ira de pair avec la mise en place d'un nouveau régime d'accumulation.

À l'intérieur du marxisme l'on retrouve généralement deux conceptions opposées de la crise fiscale. Primo, la tendance à la crise fiscale serait inhérente au développement intense des contradictions entre les dépenses de l'État et ses revenus. La crise économique ne fait alors qu'accentuer la crise

31. Le déficit du gouvernement central est passé de 3,8\$ milliards en 1975 à plus de $10 \$$ milliards annuellement depuis 1978 (plus de $20 \$$ milliards de déficit sont prévus pour la présente année). L. Pratt et J. Laxer ont démontré qu'un des objectifs du PEN était d'augmenter la part des revenus de l'exploitation du pétrole et du gaz allant au gouvernement central. De même, les nouveaux accords fiscaux et la restructuration de l'État providence peuvent être perçus comme ayant pour objectif de diminuer les dépenses du gouvernement fédéral.

Sur les accords fiscaux, en plus du document cité à la note 18, voir le projet de loi C-97 adopté par la Chambre des communes le 5 avril 1982.

32. Pour les tenants du monétarisme l'accroissement de l'interventionnisme étatique faussa le jeu du libre marché, ce qui amena la présente crise. L'objectif du monétarisme est en fait de permettre une restructuration du régime d'accumulation en conformité avec la NDIT; voir M. J. Hiscox, "Le monétarisme en actes», Critiques de l'économie politique, no 18, janvier-mars 1982, pp. 3-22. 
fiscale ${ }^{33}$. Secundo, la crise fiscale serait tout bonnement le résultat de la crise économique. L'augmentation du chômage et le ralentissement général de l'économie ont pour résultat d'augmenter les dépenses de l'État (nécessité d'augmenter les subventions et concessions fiscales aux entreprises, hausse des coûts de l'assurance-chômage, du bien-être...) et de diminuer ses revenus (baisse de revenu de l'impôt personnel, difficulté de taxer les compagnies, baisse de rendement des taxes reliées à la consommation...) $)^{34}$.

Chacun de ces deux types d'analyse comporte un avantage. Dans le premier cas, l'accent est remis sur la lutte des classes - bourgeoisie/travailleurs - pour analyser l'État, ou plus précisément le caractère contradictoire de l'État à travers le processus budgétaire. Dans le second cas, les rapports entre l'État et le régime d'accumulation sont placés au cœur de la recherche. L'État «obéit» à la logique du capital. Même l'étude de la crise fiscale nous renvoie au débat entre les tenants de «l'autonomie relative» et ceux du déterminisme des rapports sociaux de production.

Sans reprendre l'ensemble des critiques qui ont déjà été adressées aux courants structuralistes et économicistes, il importe de mentionner certains problèmes reliés à ces courants dans le but de faciliter la présentation de notre conception de la crise fiscale.

33. Ceci est la base de l'analyse de J. O'Connor (1973), The Fiscal Crisis of the State, St. Martin Press; (1979), "Some Reflective Critisicms on Mosley's 'Critical Reflections on the Fiscal Crisis of the State' ", Review of Radical Political Economics, LL, 3, Fall.

34. Ce type d'analyse peut-être retrouvé dans: P. Bullock et D. Yaffe (1975), "Inflation, the Crisis and the Post-War Boom", Revolutionary Communist, 3/4, Novembre, pp. 5-46. D. Yaffe et P. Bullock (1978), "The State and the Capitalist Crisis ", Revolutionary Communist Group.

Pour une analyse similaire tentant de démontrer que la crise du capital exige l'abandon de l'État providence, voir: CSE State Group (1979), Struggle over the State, London, CSE Books. 
Le principal problème dans l'analyse d'O'Connor réside dans le fait que l'augmentation des dépenses de l'État, donc l'accroissement de son interventionnisme, est vue comme largement autonome du procès d'accumulation ${ }^{35}$. De ceci se dégage que la sphère étatique et la crise fiscale ont une logique indépendante du procès de production et sont le résultat de la lutte des classes. Malgré toutes les précautions de l'auteur, on n'est pas très loin de l'État neutre, où la direction prise par ce dernier ne se présente que comme le résultat de la lutte des classes au niveau politique.

Pour ce qui est du courant économiciste, il nous semble incapable d'analyser en profondeur la crise fiscale. Tout ce que l'on peut en dégager c'est que l'interventionnisme de l'État s'est développé parce qu'il répondait aux besoins du capital et que la présente crise économique l'oblige à une coupe sombre dans ses dépenses improductives. Conception réactive de l'État capitaliste.

Selon nous, l'accroissement du rôle de l'État dans l'économie doit-être analysé, comme nous l'avons déjà mentionné, en rapport avec la mise en place du régime d'accumulation intensive et du rapport salarial fordiste, d'une part, et en rapport avec les luttes des travailleurs aux niveaux économique et politique, d'autre part. L'on pourrait conclure d'une telle formulation, que notre analyse ne consiste qu'en une juxtaposition des deux courants précédemment discutés. Tel n'est pourtant pas le cas. S'il est vrai que l'intervention de l'État s'est accentuée, surtout depuis la deuxième guerre mondiale, pour répondre aux besoins de l'accumulation ceci ne s'est fait qu'à partir de la mise en place d'un type nouveau de rapport salarial, qui implique dans son essence la lutte entre le capital et le travail.

35. J. O'Connor (1973) et (1979) op. cit., et (1981), "The Fiscal Crisis of the State Revisited», Kapitalistate, 9, pp. 41-61. 
De plus, si l'interventionnisme étatique dans les années 50 et la première moitié des années 60 n'a pas suscité d'oppositions vives et soutenues ni de la part du mouvement ouvrier ni de celle de la bourgeoisie, c'est parce que cet interventionnisme répondait, partiellement du moins, aux intérêts des deux classes fondamentales ${ }^{36}$. Ceci en permettant le maintien de l'accumulation et l'amélioration des conditions de vie des travailleurs.

Comment expliquer alors qu'au cours des années 70 l'intervention de l'État débouche dans la plupart des formations sociales capitalistes avancées sur une crise fiscale des gouvernements? À partir de la problématique esquissée ci-haut la crise fiscale doir-être diagnostiquée à la fois comme le résultat de la crise du régime d'accumulation - en ce sens elle en est un aspect - et comme un révélateur de l'historicité de l'État interventionniste. En effet, la crise fiscale démontre l'impossibilité de maintenir un type de rapport salarial qui n'est plus en accord avec un régime d'accumulation et même qui devient une entrave à son dépassement.

$\mathrm{Ce}$ n'est point la crise fiscale qui remet en question le rapport salarial fordiste, mais plutôt c'est ce dernier qui est devenu obsolète avec la fin de la phase expansionniste. Il constitue un élément de la crise du régime d'accumulation intensive et son maintien ne fera qu'accentuer la crise fiscale et bloquer l'émergence d'un nouveau régime d'accumulation capitaliste.

36. Trois études sur l'État providence en Grande-Bretagne démontrent comment les politiques mises en place par l'État répondaient aux besoins du capital et aux demandes des travailleurs. CSE State Group (1979), op. cit.; N. Ginsburg (1979), Class, Capital and Social Policy, Macmillan; I. Gough (1979), The Political Economy of the Welfare State, Macmillan. 


\section{Conclusion}

Les travailleurs et les masses populaires sont ceux qui ont le plus à perdre dans les restructurations en cours. Avec les tentatives de modification du rapport salarial fordiste, c'est l'amélioration et même le maintien à leur niveau actuel des conditions de vie de ces classes qui sont menacés. Il est en effet impensable qu'une transformation de l'économie canadienne en économie de plus en plus spécialisée et de plus en plus extravertie puisse permettre la continuité de l'État-providence et l'amélioration des conditions de vie de la population en général. De plus, la centralisation de l'État fédératif canadien et la concentration des pouvoirs ne pourra qu'approfondir le caractère non démocratique de l'État ${ }^{37}$.

Afin d'éviter une telle sortie de crise et la marginalisation d'une partie importante de la population, le mouvement ouvrier canadien doit proposer une solution de rechange globale préconisant des politiques touchant l'ensemble des classes de travailleurs et populaires (et non pas négocier par secteur ou par groupe de travailleurs) et chercher à démontrer que seul un changement de société peut garantir que l'État soit respectueux des besoins et intérêts des masses populaires et non de ceux du capital. Le mouvement ouvrier doit également rompre avec les analyses keynésiennes de la crise fiscale pour être en mesure non seulement de comprendre son caractère relatif mais aussi pour dévoiler les objectifs réels qui sous-tendent le débat sur la

37. Pour une analyse de l'instauration de l'État disciplinaire au Québec, cf. C. Levasseur (1980), «De l'État-Providence à l'État disciplinaire», in G. Bergeron, R. Pelletier dir., L'État du Québec en devenir, Boréal Express, Montréal, pp. 285-328. Selon C. Levasseur une régulation de plus en plus disciplinaire des rapports sociaux est essentielle pour le redémarrage de l'accumulation du capital. Avec le rôle primordial que joue le niveau fédéral de l'État canadien dans l'accumulation et la répression il nous apparaît évident que le gouvernement fédéral cherchera à accompagner la centralisation de transformations au niveau de ses fonctions historiques. 
crise fiscale. Affirmer qu'il faut accroître les interventions de l'État pour solutionner la crise doit viser à démasquer les liens entre l'État capitaliste et l'accumulation et non pas servir à reproduire l'idéologie de l'État «re-distributeur». Si le mouvement ouvrier faillit à son rôle, les conséquences de la crise actuelle résulteront en une importante défaite pour les travailleurs, la relance du procès de production de la plus-value et l'établissement d'un nouveau rapport salarial s'accompagnant d'une transformation de l'État, ou tout au moins le rétrécissement du rapport salarial existant. 